\title{
Nonuniqueness Results for X-Ray Problems with Point Sources*
}

\section{Ulrich Brehm}

Fachbereich Mathematik, Technische Universität Berlin, 1000 Berlin 12, Federal Republic of Germany

brehm@willmore.math.tu-berlin.de

\begin{abstract}
Let $X \subseteq \mathbb{R}^{d}(d \geq 2)$ be any finite set with no three points on a line. Then we construct explicitly families of bounded Borel sets which are not uniquely determined by their X-ray images from the points in $X$. This result is in sharp contrast to the known uniqueness results for convex sets (Hammer's X-ray problem).
\end{abstract}

\section{Introduction}

Let $f: \Omega \rightarrow \mathbb{R}$ be an integrable function, where $\Omega \subseteq \mathbb{R}^{d}$ is a bounded open set, $\Omega \neq \varnothing\left(f \in L_{0}^{1}(\Omega)\right)$. Then the divergent beam X-ray transform of $f$ from a source point $p \in \mathbb{R}^{d}$, or X-ray image of $f$ from $p$, is the function $\mathscr{D}_{p} f: S^{d-1} \rightarrow \mathbb{R}$ defined by

$$
\mathscr{D}_{p} f(x)=\int_{0}^{\infty} f(p+t x) d t .
$$

Physically $f$ is regarded as the density function of the object X-rayed, so that $\mathscr{D}_{p} f(x)$ is the total mass of the object along the half-line with source $p$ and direction $x$.

One of the basic problems is the question: under which conditions can $f$ be uniquely reconstructed from some of its X-ray images $\mathscr{D}_{p} f$ ? Because of their practical relevance $X$-ray problems received wide attention. We give [6] as a general reference. In [5] it is shown (among several other results) that a function $f \in L_{0}^{1}(\Omega)$ is uniquely determined by its $\mathrm{X}$-ray images $\mathscr{D}_{p} f$ for $p \in X$, where $X \subseteq \mathbb{R}^{d}$ is any infinite set with $\mathrm{cl} X \cap \mathrm{cl}$ conv $\Omega=\varnothing$ (cl $X$ denotes the closure of $X$, conv $\Omega$ denotes the convex hull of $\Omega$ ), and that any finite set of sources outside conv $\Omega$ is not sufficient to determine $f$ uniquely.

\footnotetext{
* This research was partially supported by the Italian "Consiglio Nazionale delle Ricerche."
} 
Now we want to consider the case of (characteristic functions of) sets, i.e., X-ray images of objects of constant density. For a bounded Borel set $S$ we define its $\mathrm{X}$-ray image from $p$ as $\mathscr{D}_{p} S:=\mathscr{D}_{p} 1_{s}$, where $1_{s}$ is the characteristic function of $S$, thus

$$
\mathscr{D}_{p} S(x)=\lambda\left(\left\{p+t x \mid t \in \mathbb{R}_{+}\right\} \cap S\right),
$$

where $\lambda$ denotes the one-dimensional Lebesgue measure.

The situation for convex sets is very different from the situation for integrable functions, namely that in many cases the $\mathrm{X}$-ray images from two point sources suffice to determine a convex body uniquely and the X-ray images from four point sources (no three collinear) always determine a convex body uniquely. More precisely, Falconer [3], Volčič [8], and Gardner [4] have shown that a convex body $K \subseteq \mathbb{R}^{2}$ is uniquely determined by two X-ray images with point sources $p_{1}$, $p_{2}$ if $p_{1}, p_{2} \in$ int $K$ or if $p_{1}, p_{2} \notin$ int $K$ and $L \cap K \neq \varnothing$, where $L$ denotes the line connecting $p_{1}$ and $p_{2}$. A convex body $K \subseteq \mathbb{R}^{2}$ is uniquely determined by the X-ray images from three points $p_{1}, p_{2}, p_{3} \notin$ int $K[8]$.

Volčic pointed out that a finite set of point sources would probably not be sufficient to determine each bounded measurable set uniquely, but that explicit examples were still missing even for two point sources. In this paper we present such examples for each finite set of point sources (no three on a line). Thus the situation for bounded measurable sets is similar to that for integrable functions with bounded support, but very different from the situation for convex sets.

\section{The Main Results}

For each finite set of points (sources) $X \subseteq \mathbb{R}^{d}$ (no three collinear) we construct in Theorem 2, in a universal way, families of bounded Borel sets (bounded away from the sources) having the same $X$-ray images from the points in $X$. We can even prescribe the sets in some neighborhood $U$ of any given countable bounded set $Y$ which is bounded away from the lines connecting the sources.

In Theorem 1 we give a simpler fractal-type construction in case stronger assumptions are satisfied (in particular card $X<2^{d}$ ), see Figs. 1 and 2.

We use the following standard notations:

Let $A \subseteq \mathbb{R}^{d}$.

card $A$, int $A, \mathrm{cl} A$, bd $A$, conv $A, \mathscr{P}(A), \lambda_{d}(A)$ (for $A$ measurable) denote, respectively, the cardinality, interior, closure, boundary, convex hull, power set, Lebesgue measure of $A$.

If $A$ is a Borel set contained in some straight line, $\lambda(A)$ denotes the onedimensional Lebesgue measure of $A$.

Let $x, y \in \mathbb{R}^{d}$.

$\langle x, y\rangle$ denotes the Euclidean inner product,

$\|x\|$ denotes the Euclidean norm, and, for $x \neq y$,

$L(x, y)$ denotes the half-line through $y$ with source $x$.

Now we can state our first theorem. 
Theorem 1. Let $X \subseteq \mathbb{R}^{d}$ be a finite set and let $K \subseteq \mathbb{R}^{d}$ be a bounded Borel set such that, for all $x, y \in X$ with $x \neq y$, the following conditions hold:

$$
\begin{gathered}
(K-x) \cap(K-y)=\varnothing . \\
\frac{1}{2}(K+x) \subseteq K .
\end{gathered}
$$

Let $U:=K \backslash \bigcup_{x \in X}\left(\frac{1}{2}(K+x)\right)$ and assume

$$
\lambda_{d}(U)>0
$$

Then there are mappings $F, G: \mathscr{P}(U) \rightarrow \mathscr{P}(K)$ such that

(i) $F(U) \cap G(U)=\varnothing$.

(ii) $F(A) \cap U=A$ for all $A \subseteq U$.

(iii) $F$ and $G$ map open sets onto open sets and Borel sets onto Borel sets.

(iv) $\lambda(F(A) \cap L)=\lambda(G(A) \cap L)$ for each Borel set $A \subseteq U$ and each half-line $L$ with source $x \in X$.

(v) $F$ and $G$ preserve arbitrary unions and intersections.

(vi) $\lambda_{d}(K \backslash(F(U) \cup G(U)))=0$.

(vii) $F(A), G(A)$ are countable disjoint unions of homothetic images of $A$ (with factors $\left.2^{-v}\right)$.

Remark 1. In particular, for each Borel set $A \subseteq U$ the sets $F(A) \cup G(U \backslash A)$ and $F(U \backslash A) \cup G(A)$ are disjoint Borel sets having equal X-ray images with point sources in $X$. To see this, note that (i), (iii), (iv), and (v) imply

$$
\begin{aligned}
\lambda((F(A) \cup G(U \backslash A)) \cap L) & =\lambda(F(U) \cap L)=\lambda(G(U) \cap L) \\
& =\lambda((F(U \backslash A) \cup G(A)) \cap L)=\frac{1}{2} \lambda((F(U) \cup G(U)) \cap L) .
\end{aligned}
$$

Since $A \subseteq U$ is an arbitrary Borel set and since $(F(A) \cup G(U \backslash A)) \cap U=A$ there are uncountably many essentially different sets having the same $\mathrm{X}$-ray images.

Proof of Theorem 1 . Let $f_{x}: \mathbb{R}^{d} \rightarrow \mathbb{R}^{d}$ be defined by $f_{x}(u):=\frac{1}{2}(x+u)$ (homothety with center $x$ and factor $\frac{1}{2}$ ). For $x, y \in X, x \neq y$ the sets $f_{x}(K), f_{y}(K)$, and $U$ are clearly pairwise disjoint. This implies that $U$ and sets of the form $f_{x_{1}} \cdots f_{x_{k}}(U)$ are pairwise disjoint (where $k \geq 1, x_{1}, \ldots, x_{k} \in X$ ).

Define, for $A \subseteq U$,

$$
\begin{aligned}
F(A):=A \cup \bigcup\left\{f_{x_{1}}^{n_{1}} \cdots f_{x_{h}}^{n_{h}}(A) \mid h \geq 2 \text { even, } x_{1}, \ldots, x_{h} \in X,\right. \\
\\
\left.x_{i} \neq x_{i+1} \text { for } i=1, \ldots, h-1, n_{1}, \ldots, n_{h} \geq 1\right\} . \\
G(A):=\bigcup\left\{f_{x_{1}}^{n_{1}} \cdots f_{x_{h}}^{n_{h}}(A) \mid h \text { odd, } \begin{array}{rl}
x_{1}, & \ldots, x_{h} \in X, \\
& \left.x_{i} \neq x_{i+1} \text { for } i=1, \ldots, h-1, n_{1}, \ldots, n_{h} \geq 1\right\} .
\end{array}\right.
\end{aligned}
$$


Now $F, G$ obviously have the properties (i), (ii), (iii), (v), and (vii) claimed in the theorem.

Let $n:=\operatorname{card} X$. Then $\lambda_{d}(U)=\lambda_{d}(K)\left(1-2^{-d} n\right)$, thus $n \leq 2^{d}-1$.

$$
\lambda_{d}(F(U) \cup G(U))=\lambda_{d}(U)\left(1+n 2^{-d}+n^{2} 2^{-2 d}+\cdots\right)=\frac{\lambda_{d}(U)}{1-n 2^{-d}}=\lambda_{d}(K) .
$$

Thus (vi) holds.

Finally, let $L$ be a half-line with source $x \in X$. For any Borel set $A \subseteq K$ we have

$$
\sum_{n=1}^{\infty} \lambda\left(f_{x}^{n}(A) \cap L\right)=\sum_{n=1}^{\infty} 2^{-n} \lambda(A \cap L)=\lambda(A \cap L) .
$$

Thus for any Borel set $A \subseteq U$ we get

$$
\begin{aligned}
\lambda(F(A) \cap L)= & \lambda(A \cap L)+\sum_{\substack{h \geq 2 \text { even } \\
x_{1} \neq x}} \lambda\left(f_{x_{1}}^{n_{1}} \cdots f_{x_{h}}^{n_{h}}(A) \cap L\right) \\
& +\sum_{\substack{h \geq 2 \text { even } \\
x_{2} \neq x}} \lambda\left(f_{x}^{n_{1}} f_{x_{2}}^{n_{2}} \cdots f_{x_{h}}^{n_{h}}(A) \cap L\right) \\
& =\sum_{\substack{h \geq 0 \text { even } \\
x_{1} \neq x}} \lambda\left(f_{x}^{n_{0}} f_{x_{1}}^{n_{1}} \cdots f_{x_{h}}^{n_{h}}(A) \cap L\right)+\sum_{\substack{h \geq 2 \text { even } \\
x_{2} \neq x}} \lambda\left(f_{x_{2}}^{n_{2}} \cdots f_{x_{h}}^{n_{h}}(A) \cap L\right) \\
& =\lambda(G(A) \cap L),
\end{aligned}
$$

where all sums are taken over all expressions of the indicated form with $x_{i} \neq x_{i+1}$ for $i=1, \ldots, h-1$ and $n_{0}, \ldots, n_{h} \geq 1$. Thus (iv) holds.

\section{Remark 2.}

(a) Condition (2) is for example, satisfied for any bounded open convex set $K$ with $X \subseteq$ bd $K$.

(b) Conditions (1) (3) are, for example, satisfied for $K$ being an open cube and $X$ being the set of all vertices of $K$ except one vertex.

(c) Note that (1)-(3) imply card $X \leq 2^{d}-1$ as shown in the proof of Theorem 1.

In Fig. 1 we show the following example: $K$ is the half-open square $(0,2] \times$ $(0,2] . X$ is the set $\{(0,0),(2,0),(0,2)\}$ of three vertices, thus $U=(1,2] \times(1,2]$. In this figure $G(U)$ is the black set and $F(U)$ is the white set in the square. For any arbitrary Borel set $A \subseteq U$ we merely have to replace each of the squares from which $F(U), G(U)$ are built up canonically, by the corresponding homothetic copy of $A$ to get $F(A), G(A)$, respectively.

In Fig. 2 we show a similar example, where $K$ is an open triangle and $X$ is the set of vertices of $K$. The common boundary of $F(U)$ and $G(U)$ is a self-similar fractal set in the strict sense. It is known as the "Sierpiński triangle." It was regarded by Sierpinski [7] as a curve in which each point is a ramification point. In the theory of fractals it is a standard example, see, e.g., [1]. 


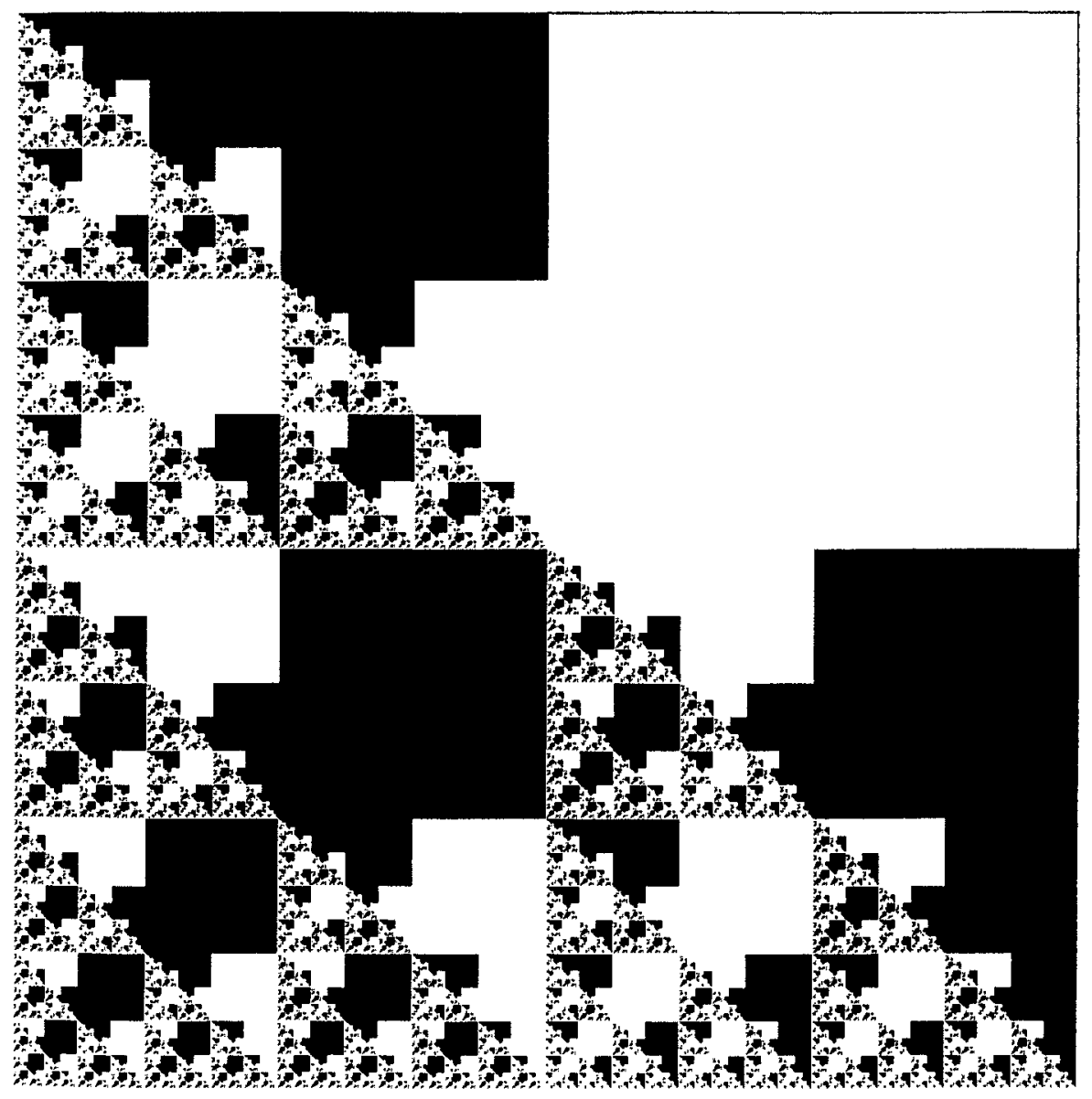

Fig. 1

As, in particular, Remark 2(c) shows, the assumptions on $K$ and $X$ in Theorem 1 are fairly strong. With a similar but more involved construction we can get essentially the same results under weaker assumptions. More precisely, for each finite set of points (sources) $X \subseteq \mathbb{R}^{d}$, no three collinear, and for each countable bounded set $Y$ which is bounded away from the lines connecting the sources, we can construct open sets $U, M$ with $Y \subseteq U \subseteq M$ and mappings $F, G: \mathscr{P}(U) \rightarrow \mathscr{P}(M)$ having essentially the same properties (namely (i)-(v)) as the ones constructed in Theorem 1. Thus, in particular, Remark 1 is also valid for Theorem 2 . In addition we can get that $M$ is bounded away from $X$.

Theorem 2. Let $X \subseteq \mathbb{R}^{d}$ (with $d \geq 2$ ) be a finite set which contains no three collinear points. Let $Y \subseteq \mathbb{R}^{d}$ be a bounded countable set such that $L(x, \tilde{x}) \cap \mathrm{cl} Y=\varnothing$ for all $x, \tilde{x} \in X$ with $x \neq \tilde{x}$. Then there exist bounded open sets $U, M \subseteq \mathbb{R}^{d}$ with 


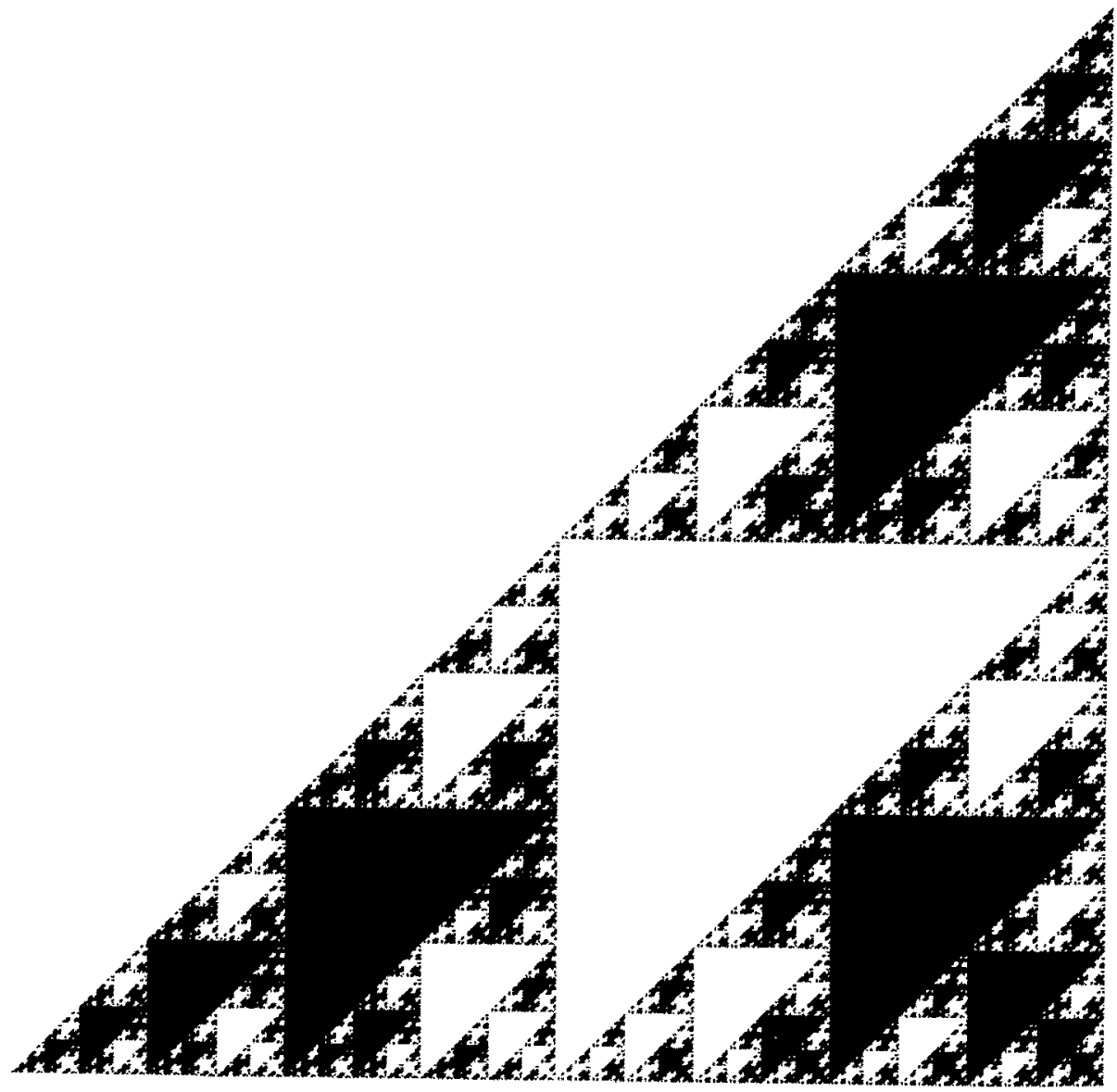

Fig. 2

$Y \subseteq U \subseteq M \subseteq \operatorname{conv}(X \cup U)$ and mappings $F, G: \mathscr{P}(U) \rightarrow \mathscr{P}(M)$ such that:

(i) $F(U) \cap G(U)=\varnothing$.

(ii) $F(A) \cap U=A$ for all $A \subseteq U$.

(iii) $F$ and $G$ map open sets onto open sets and Borel sets onto Borel sets.

(iv) $\lambda(F(A) \cap L)=\lambda(G(A) \cap L)$ for each Borel set $A \subseteq U$ and each half-line $L$ with source $x \in X$.

(v) $F$ and $G$ preserve arbitrary unions and intersections.

(vi) $X \cap \mathrm{cl} M=\varnothing$.

Proof. We first give the basic idea of our construction, which is quite similar to the proof of Theorem 1.

We construct an open set $U \subseteq \mathbb{R}^{d}$ with $Y \subseteq U$ and $X \cap \mathrm{cl} U=\varnothing$ and a family of pairwise disjoint open sets $M(x) \subseteq$ int $\operatorname{conv}(X \cup U)$ for $x \in X$, such that $M(x) \cap U=\varnothing$ and $X \cap \mathrm{cl} M(x)=\varnothing$, and a family of injective open mappings 
$f_{x}: M \backslash M(x) \rightarrow M(x)$, where $M:=U \cup \bigcup\{M(x) \mid x \in X\}$, such that, for each Borel set $A \subseteq M \backslash M(x)$ and each half-line $L$ with source $x \in X$, we have

$$
\lambda(L \cap A)=\lambda\left(L \cap f_{x}(A)\right)
$$

Now let us assume that we have already constructed $U, M(x), f_{x}$ for $x \in X$. We then show how to construct $F, G$ with the properties we want. We define three mappings:

$$
\begin{gathered}
H: \mathscr{P}(M) \rightarrow \mathscr{P}(M), \\
F: \mathscr{P}(M) \rightarrow \mathscr{P}(M), \\
G: \mathscr{P}(M) \rightarrow \mathscr{P}(M),
\end{gathered}
$$

by

$$
\begin{aligned}
H(A) & :=\left\{f_{x}(a) \mid x \in X, a \in A \backslash M(x)\right\}, \\
F(A) & :=\bigcup_{n=0}^{\infty} H^{2 n}(A), \\
G & :=H \circ F .
\end{aligned}
$$

Let $A \subseteq M$ be a Borel set with $A \cap M(s)=\varnothing$ and let $L$ be a half-line with source $s \in X$.

$$
\lambda(L \cap G(A))=\lambda(L \cap F(A))
$$

because

$$
\begin{aligned}
\lambda(L \cap G(A))=\lambda(L \cap H F(A)) & \stackrel{1}{=} \sum_{x \in x} \lambda\left(L \cap f_{x}(F(A) \backslash M(x))\right) \\
& =\lambda\left(L \cap f_{s}(F(A) \backslash M(s))\right)+\sum_{x \in X \backslash\{s\}} \lambda\left(L \cap f_{x}(F(A) \backslash M(x))\right) \\
& \stackrel{2}{=} \lambda(L \cap F(A) \backslash M(s))+\sum_{x \in X \backslash\{s\}} \lambda\left(L \cap f_{s} f_{x}(F(A) \backslash M(x))\right) \\
& \stackrel{3}{=} \lambda(L \cap F(A) \backslash M(s))+\lambda(L \cap F(A) \cap M(s)) \\
& =\lambda(L \cap F(A)) .
\end{aligned}
$$

${ }^{1}$ Because the $M(x)$ for $x \in X$ are pairwise disjoint

${ }^{2}$ Because of (4)

${ }^{3}$ Because

$$
F(A) \cap M(s)=f_{s}(H F(A) \backslash M(s)) \cup(A \cap M(s))=\bigcup_{x \in X \backslash s\}} f_{s} f_{x}(F(A) \backslash M(x))
$$

and using the facts that the $M(x)$ for $x \in X$ are pairwise disjoint and that $f_{s}$ is injective. 
$H$ preserves arbitrary unions and intersections (of nonempty families of sets) because the $f_{x}: M \backslash M(x) \rightarrow M(x)$ are injective and the $M(x)$ are pairwise disjoint. $U \cap H^{n}(U)=\varnothing$ if $n \geq 1$ because $U \cap M(x)=\varnothing$ for all $x \in X$. Thus $H^{m}(U) \cap H^{n}(U)=\varnothing$ if $m \neq n$. Thus

$$
F(U) \cap G(U)=F(U) \cap H F(U)=\varnothing .
$$

Now it is easy to see that $F, G$ have the desired properties (i), (ii), (v), (iii), (vi) using the fact that the $f_{x}$ are open, injective mappings. Thus the restrictions of $F$ and $G$ to $\mathscr{P}(U)$ also have these properties and in addition (iv) because $U \cap M(s)=\varnothing$ for all $s \in X$ and we can apply (5). These restricted mappings are exactly the mappings we seek.

To finish the proof it remains to construct $U, M(x), f_{x}: M \backslash M(x) \rightarrow M(x), x \in X$, with the properties assumed at the begining of the proof. Assume card $X \geq 2$, card $Y=\aleph_{0}$; otherwise merely enlarge $X$ and $Y$.

For the construction of $U$ and $M(x)$ we have first to define some numbers and sets. We define $n:=$ card $X$, thus $n \geq 2$ and

$$
b:=\min \{\|x-z\| x \in X, z \in X \cup \mathrm{dl} Y, x \neq z\}
$$

$b$ is the minimal distance between different points in $X$ and $X \cup \mathrm{cl} Y$.

$\alpha:=\min \left\{\arccos \frac{\left|\left\langle x_{1}-x_{2}, x_{1}-z\right\rangle\right|}{\left\|x_{1}-x_{2}\right\|\left\|x_{1}-z\right\|} \mid x_{1}, x_{2} \in X, z \in X \cup \mathrm{cl} Y, \operatorname{card}\left\{x_{1}, x_{2}, z\right\}=3\right\}$.

$\alpha$ is equal to the minimum of all angles between pairs of half-lines $L\left(x_{1}, x_{2}\right)$ and $L\left(x_{1}, z\right)$, where $x_{1}, x_{2} \in X, z \in X \cup$ cl $Y$ are three different points. $\alpha>0$ because $L\left(x_{1}, x_{2}\right) \cap \mathrm{cl} Y=\varnothing$ for all $x_{1}, x_{2} \in X$ with $x_{1} \neq x_{2}$.

$$
\begin{aligned}
& r:=\frac{b \sin \alpha}{10 n+4}, \\
& \beta:=\arcsin \left(\frac{3.1 r}{b}\right) .
\end{aligned}
$$

For $x, z \in \mathbb{R}^{d}$ with $x \neq z$ and $t \in \mathbb{R}$ define

$$
\begin{array}{r}
H(x, z, t):=\left\{w \in \mathbb{R}^{d} \mid\langle w-x, z-x\rangle=t\|z-x\|\right\}, \\
H_{+}(x, z, t):=\left\{w \in \mathbb{R}^{d} \mid\langle w-x, z-x\rangle>t\|z-x\|\right\}, \\
H_{-}(x, z, t):=\left\{w \in \mathbb{R}^{d} \mid\langle w-x, z-x\rangle\langle t\|z-x\|\} .\right.
\end{array}
$$

$H(x, z, t)$ is the hyperplane orthogonal to $x-z$ at distance $t$ from $x$ in the direction to $z$, i.e., through $x+t(z-x) /\|z-x\| . H_{+}(x, z, t)$ and $H_{-}(x, z, t)$ are the open half-spaces with boundary $H(x, z, t)$. 
For $x, z \in \mathbb{R}^{d}$ with $x \neq z$ and $\gamma \in(0, \pi)$ define

$$
C(x, z, y):=\left\{w \in \mathbb{R}^{d} \mid\langle z-x, w-x\rangle>\|z-x\|\|w-x\| \cos \gamma\right\} .
$$

$C(x, z, \gamma)$ is the open symmetric cone with apex $x$, axis $x z$, and angle $\gamma$ to the axis.

For all $x \in X, z \in X \backslash\{x\}$ define

$$
M(x, z):=C(x, z, \beta) \cap \mathrm{H}_{+}(x, z, r) \cap H_{-}(x, z, 3 r) \quad \text { and } \quad M(x, x):=\varnothing
$$

We show $M(x, z)$ in the top part of Fig. 5. Until (19) we show that the $M(x, z)$ are pairwise disjoint.

Equation (8) and $n \geq 2$ imply

$$
r \leq \frac{b}{24} \sin \alpha<\frac{b}{24}
$$

With (9) this implies that $\beta=\arcsin (3.1 r / b)<\arcsin (0.13)$ thus

$$
\frac{3}{\cos \beta}<3.1 \text {. }
$$

Now (12), (14), (6), and (9) imply, for all $x, \tilde{x}, z \in X$ with $x \neq \tilde{x}$,

$$
M(x, z) \subseteq B_{3.1 r}(x) \backslash B_{r}(x) \subseteq B_{3,1 r}(x) \subseteq C(\tilde{x}, x, \beta),
$$

where $B_{r}(x)$ denotes the open ball with center $x$ and radius $r$.

Equations (15), (12), and (13) imply, for $x, \tilde{x}, z, \tilde{z} \in X$,

$$
M(x, z) \cap M(\tilde{x}, \tilde{z})=\varnothing \quad \text { if } \quad x \neq \tilde{x} .
$$

Equations (13) and (9) imply $\sin \beta<0.13 \sin \alpha$ and $0<\beta<\pi / 2$, thus

$$
\beta<0.13 \alpha<\frac{\alpha}{2}
$$

using the convexity of $\sin$ on $[0, \pi]$.

Equations (17), (7), and (12) imply

$$
\begin{array}{r}
M(x, z) \cap M(x, \tilde{z}) \subseteq C(x, z, \beta) \cap C(x, \tilde{z}, \beta)=\varnothing \\
\text { for all } x, z, \tilde{z} \in X \text { with } z \neq \tilde{z} .
\end{array}
$$

Equations (12), (18), (16), and (15) show that

the $M(x, z), x, z \in X$, are pairwise disjoint open sets with $X \cap \operatorname{cl}(M(x, z))=\varnothing$. 
Now we define the bounded open set $U \supseteq Y$. Let $Y=\left\{y_{m} \mid m \in \mathbb{N}\right\}$ and $X=$ $\left\{x_{1}, \ldots, x_{n}\right\}$. We define recursively open sets $V_{m}$ by $V_{1}:=B_{r / 2}\left(y_{1}\right)$; if $V_{1}, \ldots, V_{m-1}$ are defined we define:

$$
V_{m}:=\varnothing \quad \text { if } \quad y_{m} \in \bigcup_{i=1}^{m-1} V_{i}
$$

otherwise $V_{m}:=B_{s_{m}}\left(y_{m}\right)$ with $0<s_{m} \leq 2^{-m} r$ and such that

$$
V_{m} \cap \bigcup_{i=1}^{m-1} V_{i}=\varnothing \quad \text { and } \quad Y \cap b d V_{m}=\varnothing \text {. }
$$

Let

$$
\begin{aligned}
& U:=\bigcup\left\{V_{m} \mid m \in \mathbb{N}\right\}, \\
& \beta_{m}:=\arcsin \frac{2^{-m_{r}}}{b} .
\end{aligned}
$$

For $m \in \mathbb{N}, i \in\{1, \ldots, n\}$ define

$$
W_{i m}:=C\left(x_{i}, y_{m}, \beta_{m}\right) \cap B_{r\left(3-2^{1-m}\right)}\left(x_{i}\right) \backslash B_{r\left(3-2^{2-m}\right)}\left(x_{i}\right) .
$$

By construction and (6), (13), (15) we have

the $V_{m}$ and $W_{i m}$ are pairwise disjoint open sets,

$$
Y \subseteq U, \quad U \cap M(x, z)=\varnothing, \quad X \cap \mathrm{cl} V_{m}=X \cap \mathrm{cl} W_{i m}=\varnothing
$$

and

$$
W_{i m} \cap M\left(x_{j}, z\right)=\varnothing .
$$

The last assertion follows from $W_{\mathrm{im}} \cap M\left(x_{j}, z\right) \subseteq B_{3 \mathrm{r}}\left(x_{i}\right) \cap B_{3.1 \mathrm{r}}\left(x_{j}\right)=\varnothing$ for $i \neq j$ and from $\beta_{m}<\beta<\alpha / 2$ as in (18) for $i=j$.

Let

$$
K:=\text { int } \operatorname{conv}(X \cup U)
$$

For $i \in\{1, \ldots, n\}$ define

$$
\begin{aligned}
M\left(x_{i}\right) & :=\left(\bigcup\left\{M\left(x_{i}, z\right) \mid z \in X\right\} \cup \bigcup\left\{W_{i m} \mid m \in \mathbb{N}\right\}\right) \cap K, \\
M & :=\tilde{U} \cup \bigcup\{M(x) \mid x \in X\} .
\end{aligned}
$$


With (19) and (24) we have all the desired properties of $U, M(x), x \in X$, namely that $U \subseteq \mathbb{R}^{d}$ is an open set with $Y \subseteq U$ and $X \cap \mathrm{cl} U=\varnothing$ and that the $M(x)$ are pairwise disjoint open sets with $M(x) \subseteq$ int $\operatorname{conv}(X \cup U), X \cap \mathrm{cl} M(x)=\varnothing$, and $M(x) \cap U=\varnothing$. It remains to construct the $f_{x_{i}}$.

For any pair of hyperplanes $H_{1}, H_{2} \subseteq \mathbb{R}^{d}$ and any $v \in \mathbb{R}^{d} \backslash\left(H_{1} \cup H_{2}\right)$ we define a mapping $\varphi_{v H_{1} H_{2}}: \mathbb{R}^{d} \backslash\left(\tilde{H}_{1} \cup \tilde{H}_{2}\right) \rightarrow \mathbb{R}^{d}$, where $\tilde{H}_{1}, \tilde{H}_{2}$ are the hyperplanes parallel to $H_{1}, H_{2}$ through $v$, as

$$
\varphi_{v H_{1} H_{2}}(w)=w-\left(v_{2}-v_{1}\right)
$$

where $v_{i}$ is the intersection point of $H_{i}$ with the line through $v$ and $w(i=1,2)$.

Alternatively, $\varphi_{v H_{1} H_{2}}$ is characterized by the following properties:

$\varphi_{v H_{1} H_{2}}\left(H_{2}\right)=H_{1}$ and the restriction of $\varphi_{v H_{1} H_{2}}$ to any line through $v$, which is not parallel to $H_{1}$ or $H_{2}$, is a translation, mapping that line into itself.

From (29) it is obvious that

$\varphi_{v H_{1} H_{2}}$ restricted to $\left(\mathbb{R}^{d} \backslash\left(\tilde{H}_{1} \cup \tilde{H}_{2}\right)\right) \backslash \varphi_{v H_{1} H_{2}}^{-1}[\{v\}]$ is one-to-one, open, and continuous

and, for each half-line $L$ with source $v$, not parallel to $H_{1}$ or $H_{2}$ and for each Borel set $A \subseteq \mathbb{R}^{d}$ with $\varphi_{\mathrm{vH}} \mathrm{H}_{2}(L \cap A) \subseteq L$ we have

$$
\lambda(L \cap A)=\lambda\left(L \cap \varphi_{v H_{1} H_{2}}(A)\right)
$$

For three affinely independent points $x, y, z \in \mathbb{R}^{d}$ let

$J(x, y, z)$ denote the hyperplane through $y$ and $z$ which meets the plane $x, y, z$ orthogonally. If $d=2$, then $J(x, y, z)$ merely denotes the line through $y$ and $z$.

Obviously

$$
\varphi_{x H(x, y, s) H(y, x, r)}=\varphi_{x H(x, y, s+r) H(y, x, 0)} .
$$

We define mappings for $x, y, z \in \mathbb{R}^{d}, s \in \mathbb{R}$ :

$$
\begin{gathered}
\psi_{(x, y, s)}:=\varphi_{x H(x, y, x) H(y, x, 0)}, \\
\eta_{(x, y, z, s)}:=\varphi_{x H(x, y, s) J(x, y, z)} .
\end{gathered}
$$


Now we can define, for $x \in X$, the desired mappings $f_{x}: M \backslash M(x) \rightarrow M(x)$ by

$$
\begin{aligned}
& f_{x}(w):=\psi_{(x, z, 4 r)}(w) \quad \text { if } \quad w \in M(z, x) \\
& :=\psi_{\left(x, y_{m}, s\right)}(w) \quad \text { if } \quad w \in V_{m} \text { with } s=3 r\left(1-2^{-m}\right) \text {, } \\
& :=\eta_{\left(x, z, x_{k}, s\right)}(w) \quad \text { if } \quad w \in M\left(z, x_{k}\right) \text { with } s=r+r(2 k-1) / n \text {, } \\
& :=\eta_{\left\{x, z, y_{m}, s\right)}(w) \quad \text { if } w \in W_{j m} \text { and } x=x_{i} \text { and } z=x_{j} \\
& \text { with } s=\left(2 i-2^{-m} \cdot 3\right) r / n \text {, }
\end{aligned}
$$

where $x, z, x_{k} \in X$ are three different points, $w \in M \backslash M(x)$, and $m \in \mathbb{N}$.

$f_{x}$ is well-defined because $M \backslash M(x)$ is contained in the disjoint union of the open sets of the form $M(z, x), V_{m}, M\left(z, x_{k}\right), W_{j m}$ according to (19), (24), (26), and (27). We have to show that the images of these sets under $f_{x}$ are indeed contained in $M(x)$ and that they are pairwise disjoint. When we have shown this, then (30), (31), (34), (35) imply that the $f_{x_{i}}$ are injective open mappings satisfying (4), finishing the proof.

Using (15) and (12) it is easy to check, for $x, z \in X$, that

$$
f_{x}(M(z, x)) \subseteq M(x, z)
$$

Next we show

$$
f_{x_{1}}\left(V_{m}\right) \subseteq W_{i m}
$$

Proof. If $V_{m}=\varnothing$ there is nothing to show. Thus assume $V_{m}=B_{s_{m}}\left(y_{m}\right)$. $V_{m} \subseteq C\left(x_{i}, y_{m}, \beta_{m}\right)$, thus $f_{x_{i}}\left(V_{m}\right) \subseteq C\left(x_{i}, y_{m}, \beta_{m}\right)$. Let $a:=3 r\left(1-2^{-m}\right)$. Then

$$
\psi_{\left(x_{i}, y_{m}, a\right)}\left(B_{s_{m}}\left(y_{m}\right)\right) \subseteq B_{s_{m}}\left(x_{i}+a \frac{y_{m}-x_{i}}{\left\|y_{m}-x_{i}\right\|}\right) \subseteq B_{a+s_{m}}\left(x_{i}\right) \backslash B_{a-s_{m}}\left(x_{i}\right) \quad \text { (see Fig. 3). }
$$

Now $s_{m} \leq 2^{-m} r$ and with (23) this shows (38).

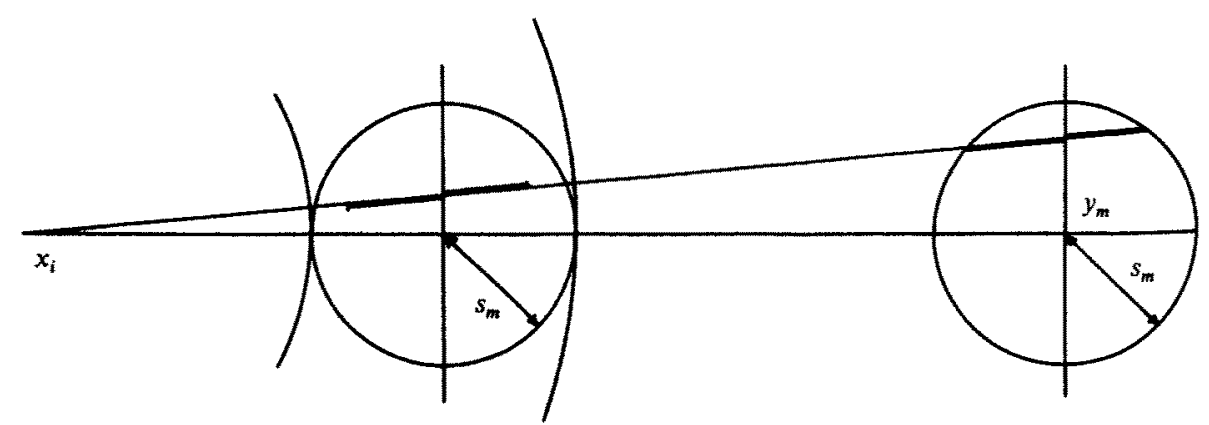

Fig. 3 


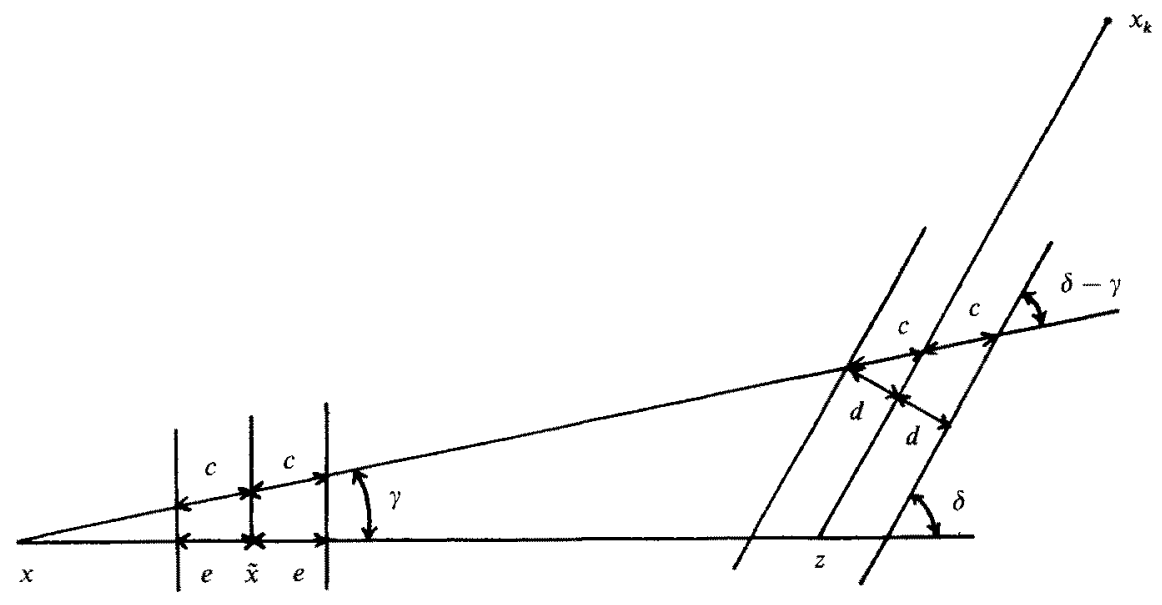

Fig. 4

We show, for $x, z, x_{k} \in X$ with $\operatorname{card}\left\{x, z, x_{k}\right\}=3$, that

$$
f_{x}\left(M\left(z, x_{k}\right)\right) \subseteq H_{+}\left(x, z, r+\frac{2(k-1) r}{n}\right) \cap H_{-}\left(x, z, r+\frac{2 k r}{n}\right)
$$

Proof. From (12) and Fig. 5 it is clear that $M\left(z, x_{k}\right)$ is contained in the "slice" between the two hyperplanes parallel to $J\left(x, z, x_{k}\right)$ at distance $d:=3 r \tan \beta$. Let $w \in M\left(z, x_{k}\right)$ and

$$
\tilde{x}=x+(2 k-1) \frac{r}{n} \cdot \frac{(z-x)}{\|z-x\|}
$$

Now for the lengths and angles in Fig. 4 we see that $c=d / \sin (\delta-\gamma), e=c \cos \gamma$, thus

$$
e=\frac{3 r \tan \beta \cos \gamma}{\sin (\delta-\gamma)}=\frac{3 r \tan \beta}{\sin \delta-\cos \delta \tan \gamma}
$$

By the definition of $\alpha$ we have

$$
\delta \geq \alpha, \quad \delta \leq \pi-\alpha, \text { and } \quad 0<\alpha \leq \frac{\pi}{2}
$$

On the other hand, since $w \in M(z) \subseteq B_{3.1 r}(z)$ we have, with (9), (8), and (15),

$$
\sin \gamma \leq \sin \beta=\frac{3.1 r}{b}=\frac{3.1 \sin \alpha}{10 n+4}
$$


Equations (40)-(42) imply

$$
e<\frac{3 r \tan \beta}{\sin \alpha-\tan \beta}=\frac{3 r}{((10 n+4) / 3.1) \cos \beta-1},
$$

thus using (14)

$$
e<\frac{r}{n}
$$

which implies (39).

On the other hand, we get with $(15) f_{x}\left(M\left(z, x_{k}\right)\right) \subseteq C(x, z, \beta)$, thus

$$
f_{x}\left(M\left(z, x_{k}\right)\right) \subseteq M(x, z)
$$

Similarly as (39) we get, for $i \neq j$ and $m \in \mathbb{N}$,

$$
f_{x_{i}}\left(W_{j m}\right) \subseteq H_{+}\left(x_{i}, z, r+\left(2 i-2^{-m} \cdot 4\right) \frac{r}{n}\right) \cap H_{-}\left(x_{i}, z, r+\left(2 i-2^{-m} \cdot 2\right) \frac{r}{n}\right)
$$

To see this we replace $x_{k}$ by $y_{m}$ in Fig. 4 and define $z=x_{j}$ and

$$
\tilde{x}=x_{i}+\left(2 i-2^{-m} \cdot 3\right) \frac{r}{n} \cdot \frac{\left(z-x_{i}\right)}{\left\|z-x_{i}\right\|} \text { and } d:=3 r \tan \beta_{m} \text {. }
$$

As in the proof of (39) we get

$$
e<\frac{3 r \tan \beta_{m}}{\sin \alpha-\tan \beta}
$$

Now from (22), (9), and (14) we get

$$
\begin{gathered}
\sin \beta_{m}=\frac{2^{-m} r}{b}, \\
\cos \beta_{m}>\cos \beta>\frac{3}{3.1},
\end{gathered}
$$

and

$$
\tan \beta<\frac{9.61}{3} \cdot \frac{r}{b}<\frac{4 r}{b}
$$


Combining these inequalities with (46) and (8) we get

$$
e<\frac{3.1 \cdot 2^{-m} r}{10 n}<\frac{2^{-m} r}{n}
$$

proving (45).

As in (15) we get

$$
W_{j m} \subseteq B_{3.1 r}\left(x_{j}\right) \subseteq C\left(x_{i}, x_{j}, \beta\right)
$$

thus with (45)

$$
f_{x_{i}}\left(W_{j m}\right) \subseteq M\left(x_{i}, x_{j}\right)
$$

We show, for $x, z, x_{k} \in X$ with $\operatorname{card}\left\{x, z, x_{k}\right\}=3$, that

$$
f_{x}\left(M\left(z, x_{k}\right)\right) \cap f_{x}(M(z, x))=\varnothing
$$

Proof. It is easy to check (see Fig. 5) that

$$
M(z, x) \subseteq C(x, z, \gamma) \quad \text { with } \quad \gamma:=\arctan \left(\frac{3 r \tan \beta}{\|z-x\|-3 r}\right)
$$

thus $f_{x}(M(z, x)) \subseteq C(x, z, \gamma)$. It is easy to check (see Fig. 5) that

$$
C(x, z, \varepsilon) \cap C\left(z, x_{k}, \beta\right) \backslash B_{r}(z)=\varnothing,
$$

which implies

$$
C(x, z, \varepsilon) \cap f_{x}\left(M\left(z, x_{k}\right)\right)=\varnothing
$$

where $\varepsilon:=\arctan ((r \sin (\alpha-\beta)) /(\|z-x\|+r))$. Note that the angle between $L(z, x)$ and $L\left(z, x_{k}\right)$ is between $\alpha$ and $\pi-\alpha$ (in Fig. 5 we show the case that it is equal to $\alpha$ ). It remains to show that $\gamma \leq \varepsilon$.

Equations (13) and (9) imply $\sin \alpha \geq(24 / 3.1) \sin \beta$ thus with (14) we get

$$
\begin{aligned}
\sin (\alpha-\beta) & =\sin \alpha \cos \beta-\cos \alpha \sin \beta \\
& \geq\left(\frac{24}{3.1}\right)\left(\frac{3}{3.1}\right) \sin \beta-\sin \beta>6.4 \sin \beta \\
& \geq \frac{19.2}{3.1} \tan \beta>6 \tan \beta,
\end{aligned}
$$




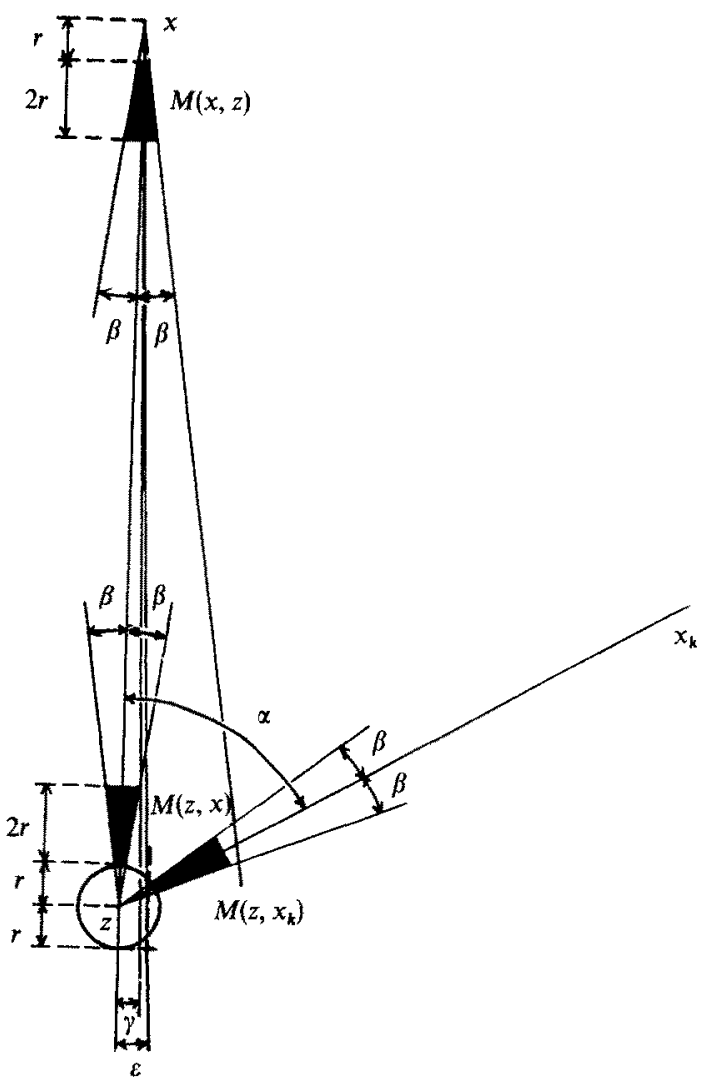

rig. 5

thus, using $\|z-x\| \geq b>24 r$,

$$
\frac{3 r \tan \beta}{\|z-x\|-3 r}<\frac{3 r \sin (\alpha-\beta)}{6(\|z-x\|-3 r)}<\frac{3 r \sin (\alpha-\beta)}{5\|z-x\|-18 r+24 r}<\frac{r \sin (\alpha-\beta)}{\|z-x\|+r} .
$$

Thus $\gamma<\varepsilon$, which shows (48).

For $x, x_{j} \in X$ with $x \neq x_{j}$ and $m \in \mathbb{N}$ we have

$$
f_{x}\left(W_{j m}\right) \cap f_{x}\left(M\left(x_{j}, x\right)\right)=\varnothing .
$$

Proof. Note that $W_{j m} \subseteq C\left(x_{j}, y_{m}, \beta\right) \backslash B_{r}\left(y_{m}\right)$. Now we can apply the proof of (48) when replacing $z$ by $x_{j}$ and $x_{k}$ by $y_{m}$.

Finally, it is obvious from definition (36) and the choice of $r$ (8) that $f_{x}(w) \in \operatorname{conv}(x, w) \backslash\{x\}$ for each $w \in M \backslash M(x)$, thus

$$
f_{x}(M \backslash M(x)) \subseteq K
$$


Now (19), (24), (37)-(39), (44), (45), and (47)-(50) imply that $f_{x}(w) \in M(x)$ for each $w \in M \backslash M(x)$ and that the images of the sets $M(z, x), V_{m}, M\left(z, x_{k}\right)$, and $W_{j m}$ are pairwise disjoint. This finishes the proof.

\section{Final Remarks}

In our constructions in Theorems 1 and 2 it was unavoidable that the closure of the sets which are constructed had points on the line segments connecting the sources. Thus even examples of compact sets in the interior of a triangle, which are not uniquely determined by the X-ray images from the vertices of the triangle, are missing. For the construction of such examples different ideas are required.

Similarly, the case of collinear points remains open.

For two point sources we have examples of finite unions of homothetic copies of polygons (bounded away from the line connecting the sources), which are not determined by their two X-ray images (see [2]).

\section{Acknowledgments}

I am grateful to Prof. Volčič for many helpful discussions and to the Italian national committee for research for supporting my stay in Trieste.

\section{References}

1. M. Barnsley, Fractals Everywhere, Academic Press, San Diego, 1988.

2. U. Brehm, Polygons which are not uniquely determined by their $\mathrm{X}$-ray images from two point sources, in preparation.

3. K. J. Falconer, X-ray problems for point sources, Proc. London Math. Soc. 46 (1983), 241-262.

4. R. J. Gardner, Symmetrals and X-rays of planar convex bodies, Arch. Math. 41 (1983), 183-189.

5. C. Hamaker, K. T. Smith, D. C. Solomon, and S. L. Wagner, The divergent beam X-ray transform, Rocky Mountain J. Math. 10 (1980), 235-283.

6. F. Natterer, The Mathematics of Computerized Tomography, Wiley and Teubner, New York and Stuttgart, 1986.

7. W. Sierpiński, Oevres choisies, Tome III, PWN Éditions sci. de Pologne, Warszawa, 1975, pp. 99-106.

8. A. Volčič, A three-point solution to Hammer's X-ray problem, J. London Math. Soc. 34 (1986), 340-359.

Received October 1, 1990, and in revised form January 23, 1991. 